\title{
Evaluation of Innovation and Entrepreneurship Ability of Computer Majors based on Neural Network Optimized by Particle Swarm Optimization
}

\author{
https://doi.org/10.3991/ijet.v16i20.26507 \\ Li Ding $\left({ }^{\bowtie}\right)$, Xuguang Chai, Fanjin Zeng \\ Hebei Vocational University of Technology and Engineering, Xingtai, China \\ $30941260 @ q q . c o m$
}

\begin{abstract}
The current evaluation index systems (EISs) of innovation and entrepreneurship (I\&E) ability are not sufficiently systematic, scientific, or practical. To solve the problem, this paper tries to evaluate the I\&E ability of computer majors, using neural networks improved by particle swarm optimization (PSO). Firstly, an EIS of 22 second-level indexes under 5 firstlevel indexes was designed to evaluate the I\&E ability of college computer majors. Next, an evaluation model was developed based on fuzzy neural network (FNN), and the corresponding training algorithm was created. Moreover, an improved PSO was introduced to optimize the FNN, and the optimization process was detailed. The proposed model was proved effective through experiments.
\end{abstract}

Keywords - computer majors, innovation and entrepreneurship (I\&E) ability, fuzzy neural network (FNN), particle swarm optimization (PSO)

\section{Introduction}

The employment situation is quite severe in current society, and college graduates are under great employment pressure [1-4], so in order to help them find suitable jobs and provide them with more employment options, colleges and universities have set various innovation and entrepreneurship training programs and education courses, in the hops of cultivating innovative and entrepreneurial talents for the country [5-8].

Studies on I\&E ability have accumulated fruitful results in recent years [9-15], for example, Su and $\mathrm{Yu}$ [16] explored the connotation and significance of the "Internet + education" model, and developed a new school-enterprise cooperation model. Louis Louw et al. [17] empirical tested the spatial spillover effects of regional economic development level on the I\&E ability of the region, they constructed a Spatial Dubin Model (SDM) and verified the significant positive spatial correlation between regional I\&E ability and regional economy. Chen [18] discussed the features and elements of college students' I\&E ability, screened the indexes, and established an EIS for it, also, the weight values of each index were given in the paper. Kim et al. [19] introduced the experiencing-type deliberate practice training into college students' entre- 
preneurship and career guidance courses, combining with the experiments on the I\&E ability of college students majoring in computer science, they proved that there're obvious differences in the students' I\&E ability before and after implementing the I\&E teaching mode based on creative problem-solving. Xu et al. [20] explored deep into the aspects of the integration of computer technology, the innovation mode, and the I\&E ability training of computer professionals. For the purpose of cultivating students' I\&E ability, Ran and Wang [21] optimized the teaching mode of computer network experimental courses. Scholar Li [22] proposed an all-around mechanism for cultivating innovative talents majoring in computer science in local colleges and universities, and integrated it into the construction of professional curriculum and the talent training mode.

Existing EISs of I\&E ability are not sufficiently systematic, scientific, or practical [23-27], therefore, this paper attempts to research the evaluation of I\&E ability of college computer majors and propose a new idea to optimize the neural network model using PSO. The second chapter of this paper constructs an EIS of I\&E ability of college computer majors containing five first-level indexes of innovation ability, professional quality, entrepreneurial resource integration, team organization and management, and entrepreneurial risk-taking. The third chapter constructs an FNN-based evaluation model and gives the corresponding training algorithm. The fourth chapter elaborates on the optimization method of the FNN optimized by an improved PSO; the last chapter uses experimental results to verify the effectiveness of the proposed model.

\section{The proposed EIS}

After fully and comprehensively considering the differences between computer majors and students of other majors, this paper designed an EIS of I\&E ability of computer majors based on a few principles such as the comprehensive principle, the scientific principle, and the operable principle, etc. The professional ability of computer majors can be described from aspects such as whether a student has obtained certificates in software-related exams, and whether the student has software patents or copyrights, etc. The practical ability of computer majors can be measured by the index of whether a student has won awards in computer skill competitions. This paper mapped the student's personal computer education experience to their career planning direction and I\&E ability, and used 5 first-level indexes (innovation ability, professional quality, entrepreneurial resource integration, team organization and management, and entrepreneurial risk-taking) to explain the main evaluation content of the I\&E ability of college students majoring in computer science, the details are given in Figure 1 .

As can be seen from the figure, the first-level index innovation ability $I E_{1}$ contains 3 second-level indexes: innovation awareness $I E_{11}$, learning ability of professional knowledge of computer technology $I E_{12}$, practical operation ability of computer technology $I E_{13}$; the first-level index professional quality $I E_{2}$ contains 8 second-level indexes: braveness $I E_{21}$, self-confidence $I E_{22}$, toughness $I E_{23}$, integrity $I E_{24}$, positivity 
$I E_{25}$, optimism $I E_{26}$, execution ability $I E_{27}$, sense of responsibility $I E_{28}$; the first-level index entrepreneurial resource integration $I E_{3}$ contains 3 second-level indexes: entrepreneurial resource requirement analysis ability $I E_{31}$, entrepreneurial resource allocation ability $I E_{32}$, entrepreneurial fund raising ability $I E_{33}$; the first-level index team organization and management $I E_{4}$ contains 4 second-level indexes: interpersonal communication ability $I E_{41}$, leadership ability $I E_{42}$, teamwork ability $I E_{43}$, team operation ability $I E_{44}$; the first-level index entrepreneurial risk-taking $I E_{5}$ contains 4 second-level indexes: computer industry entrepreneurial risk recognition ability $I E_{51}$, computer industry entrepreneurial risk resistance ability $I E_{52}$, stress resistance ability $I E_{53}$, self-regulation ability $I E_{54}$.

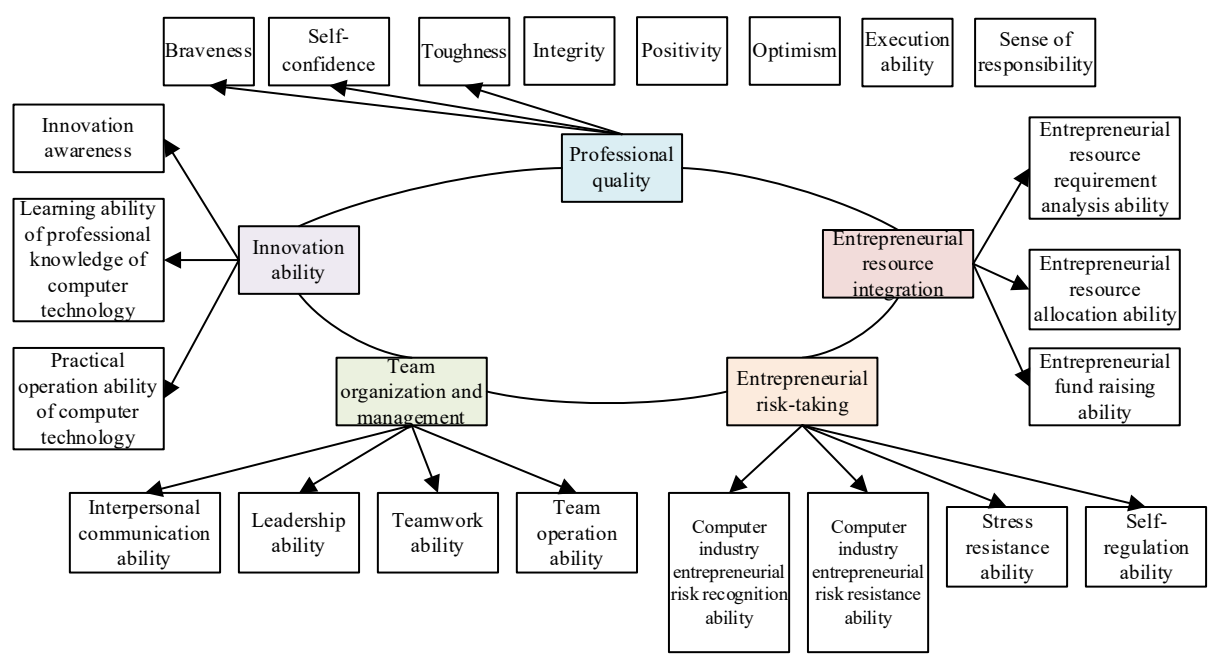

Fig. 1. Evaluation content of I\&E ability of computer majors

\section{FNN-based evaluation model and training algorithm}

To construct a FNN for evaluating the I\&E ability of computer majors, the first step was to build a neural network with multiple hidden layers for storing the fuzzy control language and sentences, then, through offline training of the network, the connection weight values were updated to realize the fuzzy reasoning of the I\&E ability of computer majors. Figure 2 shows the structure of the constructed FNN. Assuming the constructed 5-layer network has $m$ inputs and $e$ outputs, then the node number of each layer could be determined according to the following method:

For the input layer nodes of the network, their number was set to be the same as the number of evaluation index samples, respectively were $a_{l}, a_{2}, \ldots, a_{m}$, and there're $m$ nodes in total. 


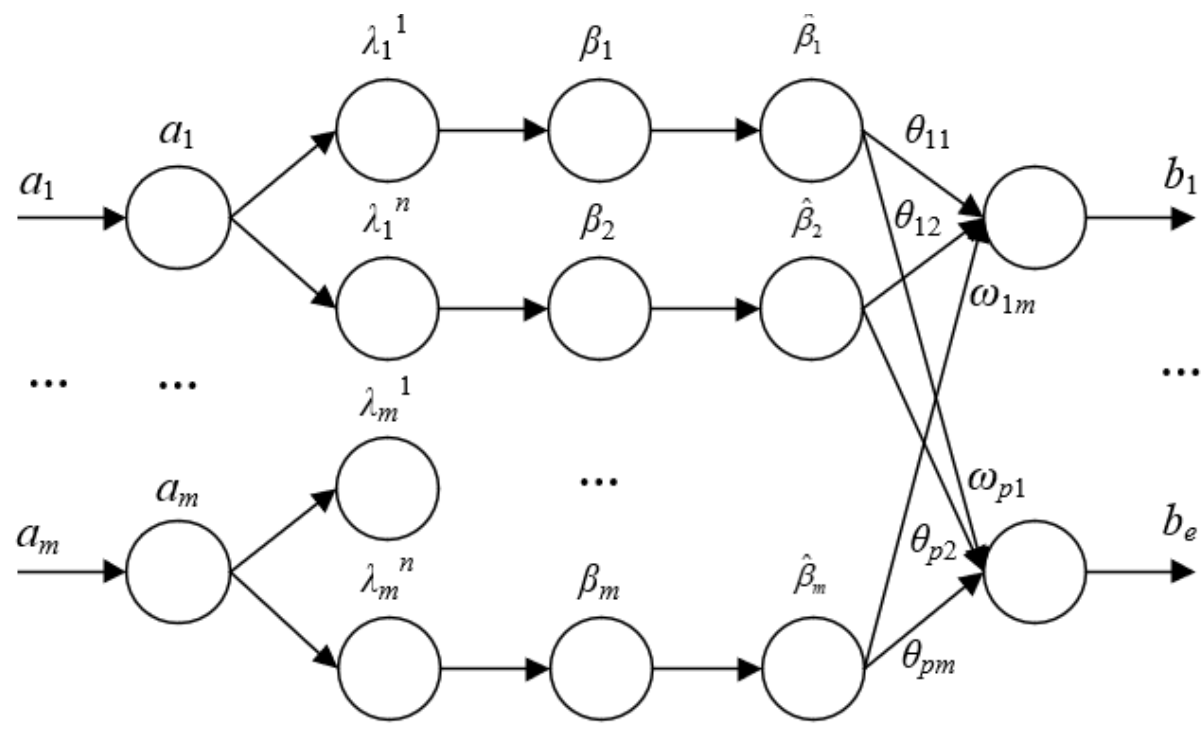

Fig. 2. Structure the constructed FNN

Assuming: every input evaluation index sample has $n$ fuzzy language variables, then, the number of neuron nodes in the fuzzy language layer of the network is $n \times m$. Since the input layer is directly connected to the fuzzy language layer, the membership degree of the fuzzy language variables can be judged according to the input value of each evaluation index sample based on the Gaussian function. Assuming: $\lambda_{i}{ }^{j}$ represents the membership degree function of the $i$-th neuron node of the input layer on the $j$-th fuzzy language variable, and $H E_{i j}$ and $\varepsilon_{i j}$ represent the central value and width of the membership degree function, then, there is:

$$
\lambda_{i}^{j}=e^{-\frac{\left(a_{i}-H E_{i j}\right)^{2}}{\varepsilon_{i j}^{2}}}
$$

For the fuzzy rule layer that is used to describe the fuzzy rules, usually, its node number is set to be the $m$ power of $n$, namely $n^{m}$. Formula 2 gives the calculation formula of the fitness degree $\beta_{j}$ of the fuzzy rules:

$$
\beta_{j}=\lambda_{1}^{i_{1}} \lambda_{2}^{i_{2}} \ldots \lambda_{m}^{i_{m}}
$$

As for the normalization layer that is used to normalize the fitness degree of the fuzzy rules, its neuron node number was set to be the same as the number of nodes in the fuzzy rule layer, namely $n^{m}$. Formula 3 gives the formula for the normalization processing of this layer:

$$
\hat{\beta}_{j}=\frac{\beta_{j}}{\sum_{i=1}^{n^{m}} \beta_{i}}
$$


The final output of the network output layer that realizes clear operations is the evaluation result of the I\&E ability of computer majors. Assuming: $\theta$ represents the connection weight of the network, then Formula 4 gives the formula for the clear operations of this layer:

$$
b=\theta \hat{\beta}
$$

The three variables can be expressed as a vector shown as Formula 5:

$$
b=\left(\begin{array}{l}
b_{1} \\
b_{2} \\
\vdots \\
b_{m}
\end{array}\right), \theta=\left(\begin{array}{cccc}
\theta_{11} & \theta_{12} & \cdots & \theta_{1 n^{m}} \\
\theta_{21} & \theta_{22} & \cdots & \theta_{2 n^{m}} \\
\vdots & \vdots & \ddots & \vdots \\
\theta_{p 1} & \theta_{p 2} & \cdots & \theta_{p n^{m}}
\end{array}\right), \hat{\beta}=\left(\begin{array}{l}
\hat{\beta}_{1} \\
\hat{\beta}_{2} \\
\vdots \\
\hat{\beta}_{n^{m}}
\end{array}\right)
$$

Since the numbers of input and output neuron nodes of the constructed FNN model were fixed, and the fuzzy language number was also a fixed value $n$, then, in the mod$\mathrm{el}$, the parameters that need to be updated via training included the connection weight $\theta$, and the central value $H E_{i j}$ and width $\varepsilon_{i j}$ of the membership degree function. The network structure of the constructed FNN model was a feedforward-type network, so it could simulate the forward propagation process of the error of BP neural network to perform iterative operations on $\theta, H E_{i j}$ and $\varepsilon_{i j}$.

First, based on the forward propagation of the input sample data, the input and output of each layer of the neural network were calculated; assuming: $g_{i}{ }^{(r)}$ and $h_{i}{ }^{(r)}$ represent the input processing and activation processing of the $i$-th neuron in the $r$-th layer of the neural network, and $a_{i}{ }^{(r)}$ represents the output of the $i$-th neuron in the $r$-th layer of the neural network, then the output and input of the input layer satisfied the following equations:

$$
g_{i}^{(1)}=a_{i}^{(0)}=a_{i}, a_{i}^{(1)}=h_{i}^{(1)}=g_{i}^{(1)}
$$

Assuming: for the $i$-th neuron, there're $n_{i}$ corresponding fuzzy language, the value range of $j$ is $\left[1, n_{i}\right]$, then the output and input of the fuzzy language layer satisfy:

$$
g_{i j}^{(2)}=-\frac{\left(a_{i}^{(1)}-H E_{i j}\right)^{2}}{\varepsilon_{i j}^{2}}, a_{i j}^{(2)}=\lambda_{1}^{j}=h_{i j}^{(2)}=e^{g_{i j}^{(2)}}=e^{-\frac{\left(a_{i}^{(1)}-H E_{i j}\right)^{2}}{\varepsilon_{i j}^{2}}}
$$

Since the number of fuzzy rule sentences was $n^{m}$ and the value range of $j$ was [1, $n^{m}$ ], the output and input of the fuzzy rule layer satisfied the following equations:

$$
g_{j}^{(3)}=\lambda_{1}^{i_{1}} \lambda_{2}^{i_{2}} \ldots \lambda_{m}^{i_{m}}, a_{j}^{(3)}=\beta_{j}=h_{j}^{(3)}=g_{j}^{(3)}
$$

The value range of $j$ of the normalization layer was also $\left[1, n^{m}\right]$, and the output and input of this layer satisfied the following equations:

$$
g_{j}^{(4)}=a_{j}^{(3)} / \sum_{i=1}^{n^{m}} a_{i}^{(3)}=\beta_{j} / \sum_{i=1}^{n^{m}} \beta_{j}, a_{j}^{(4)}=\hat{\beta}_{j}=h_{j}^{(4)}=g_{j}^{(4)}
$$


The value range of output layer $i$ was $[1, e]$, the output and input of this layer satisfied:

$$
g_{j}^{(4)}=\sum_{j=1}^{n^{m}} \theta_{i j} a_{i}^{(4)}=\sum_{j=1}^{n^{m}} \theta_{i j} \hat{\beta}_{j}, a_{j}^{(5)}=b_{i}=h_{j}^{(5)}=g_{j}^{(5)}
$$

After the evaluation of the I\&E ability of computer majors was output by the output layer, it could be compared with the expected evaluation result. The adjustment of all neural network parameters such as $\theta, H E_{i j}$ and $\varepsilon_{i j}$ was to ensure the smallest output error. Assuming: $\varphi_{i}$ represents the expected value of the $i$-th neuron in the output layer, $b_{i}$ represents the actual value of the $i$-th neuron in the output layer, $M$ represents the size of the training data, then, Formula 11 gives the function for quantifying the errors of each output neuron:

$$
D G=\frac{1}{M} \sum_{i=1}^{M}\left(\phi_{i}-b_{i}\right)
$$

The neural network training method used in this paper referred to the error forward propagation of the BP neural network, this method adjusted parameters $\theta, H E_{i j}$ and $\varepsilon_{i j}$ based on the correction of the calculation results of propagation error of each layer. Formula 12 gives the calculation formula of the output layer error:

$$
\xi_{i}^{(5)}=-\frac{\partial D G}{\partial g_{i}^{(5)}}=-\frac{\partial D G}{\partial b_{i}}=\phi_{i}-b_{i}
$$

The connection weight $\theta$ between the normalization layer and output layer neurons needs to be adjusted. Based on the propagation error of the output layer, the increment of $\theta$ can be calculated using Formula 13:

$$
\Delta \theta_{i j}=-\frac{\partial D G}{\partial \theta_{i j}}=-\frac{\partial D G}{\partial g_{i}^{(5)}} \frac{\partial g_{i}^{(5)}}{\partial \theta_{i j}}=-\xi_{i}^{(5)} a_{i}^{(4)}=-\left(\phi_{i}-b_{i}\right) \hat{\beta}_{i}
$$

Formula 14 gives the calculation formula of the error that is forwarded to the normalization layer:

$$
\xi_{j}^{(4)}=-\frac{\partial D G}{\partial g_{i}^{(4)}}=-\sum_{i=1}^{e} \frac{\partial D G}{\partial g_{i}^{(5)}} \frac{\partial g_{i}^{(5)}}{\partial h_{i}^{(4)}} \frac{\partial h_{i}^{(4)}}{\partial g_{i}^{(4)}}=\sum_{i=1}^{e} \xi_{i}^{(5)} \theta_{i j}
$$

Formula 15 gives calculation formula of the error that is forwarded to the fuzzy rule layer:

$$
\xi_{j}^{(3)}=-\frac{\partial D G}{\partial g_{i}^{(3)}}=-\sum_{i=1}^{e} \frac{\partial D G}{\partial g_{i}^{(4)}} \frac{\partial g_{i}^{(4)}}{\partial h_{i}^{(3)}} \frac{\partial h_{i}^{(3)}}{\partial g_{i}^{(3)}}=\xi_{i}^{(3)} \sum_{i=1}^{n^{m}} \beta_{i} /\left(\sum_{i=1}^{n^{m}} \beta_{i}\right)^{2}
$$

Formula 16 gives the calculation formula of the error that is forwarded to the fuzzy language layer:

$\xi_{j}^{(2)}=-\frac{\partial D G}{\partial g_{i}^{(2)}}=-\sum_{i=1}^{n^{m}} \frac{\partial D G}{\partial g_{i}^{(3)}} \frac{\partial g_{i}^{(3)}}{\partial h_{i}^{(2)}} \frac{\partial h_{i}^{(2)}}{\partial g_{i}^{(2)}}=\sum_{l=1}^{n^{m}} \xi_{i}^{(3)} e^{g_{i j}^{(2)}}=\sum_{l=1}^{n^{m}} \xi_{i}^{(3)} e^{\frac{\left(a_{i j}-H E_{i j}\right)^{2}}{\varepsilon_{i j}^{(2)}}}$ 
Considering that the central value $H E_{i j}$ and the width $\varepsilon_{i j}$ of the membership degree function were both in the fuzzy language layer, Formula 17 gives the calculation formula of the increment of $H E_{i j}$ when the error is forwarded to the fuzzy language layer:

$$
\Delta H E_{i j}=\frac{\partial D G}{\partial H E_{i j}}=\frac{\partial H E}{\partial g_{i}^{(2)}} \frac{\partial g_{i}^{(2)}}{\partial H E_{i j}}=-\xi_{j}^{(2)} \frac{2\left(a_{i j}-H E_{i j}\right)}{\varepsilon_{i j}^{(2)}}
$$

Formula 18 gives the calculation formula of the increment of width $\varepsilon_{i j}$ of the corresponding membership degree function:

$$
\Delta \varepsilon_{i j}=\frac{\partial D G}{\partial \varepsilon_{i j}}=\frac{\partial D G}{\partial g_{i}^{(2)}} \frac{\partial g_{i}^{(2)}}{\partial \varepsilon_{i j}}=-\xi_{j}^{(2)} \frac{2\left(a_{i j}-H E_{i j}\right)^{2}}{\varepsilon_{i j}^{(3)}}
$$

Formula 19 shows the formula for adjusting the connection weight $\theta$ during the training process of the neural network:

$$
\theta_{i j}(l+1)=\theta_{i j}(l)-\delta \Delta \theta_{i j}
$$

Formula 20 gives the formula for adjusting the central value $H E_{i j}$ of the membership degree function:

$$
H E_{i j}(l+1)=H E_{i j}(l)-\delta \Delta H E_{i j}
$$

Formula 21 gives the formula for adjusting the width $\varepsilon_{i j}$ of the membership degree function:

$$
\varepsilon_{i j}(l+1)=\varepsilon_{i j}(l)-\delta \Delta \varepsilon_{i j}
$$

\section{The FNN optimization method based on improved PSO}

This paper adopted an improved PSO to optimize the constructed FNN. The improved PSO realizes the balancing between global optimal value searching and local optimal value searching of the algorithm and ensures accurate optimal value searching and fast convergence by adjusting the speed of the particle swarm and the learning factors.

To improve the algorithm' ability to search for global and local optimal values, this paper introduced the inertia weight $\chi$ that affects the speed adjustment into the speed adjustment process, Formula 22 gives the formula for adjusting the speed of the particle swarm:

$$
\begin{aligned}
& w_{i j}(\phi+1)=\chi \cdot w_{i j}(\phi)+H E_{1} p_{1}\left(\operatorname{tbest}_{i j}(\phi)-c_{i j}(\phi)\right) \\
& +H E_{2} p_{2}\left(\operatorname{hbest}_{j}(\phi)-c_{i j}(\phi)\right)
\end{aligned}
$$

In fact, $\chi$ characterizes the size of the impact of particle speed at moment $\varphi$ on the speed of the particle at moment $\varphi+1$. The inertia weight $\chi$ in this paper adopted an adaptive linear adjustment strategy. The PSO needs to increase the search space of the 
particle swarm in the initial execution stage to find the location of optimal solution of the particle swarm faster, at this time, it needs to set a larger $\chi$ value. In the later execution stage, the PSO needs to enhance the particle swarm's ability to search for local optimal solution, and then find the accurate location of the optimal solution of the particle swarm faster, at this time, it needs to set a smaller $\chi$ value. Assuming: $\chi_{C}$ represents the inertia weight in the initial execution stage of the algorithm and its value range is $[0.9,1.2] ; \chi_{F}$ represents the inertia weight in the later execution stage of the algorithm and its value range is $[0.4,0.8] ; \psi$ represents the number of iteration times; $\varphi$ represents the number of current iterations; Formula 23 gives the formula for adjusting the inertia weight $\chi$ :

$$
\chi=\chi_{F}+\left(\chi_{C}-\chi_{F}\right) * \frac{(\psi-\phi)}{\psi}
$$

In the learning factors, the cognition factor describes the degree of cognition of each particle to its own position, and the social factor describes the impact of the overall position of the particle swarm to each particle. Wherein, fitting has a great impact on the individual particles, and the latter is conducive to conducting local search in the region where the optimal solution is located. In order to effectively improve the accuracy and convergence speed of the algorithm, this paper adjusted the cognition factor $H E_{1}$ and the social factor $H E_{2}$ in Formula 29. Assuming $H E_{1 C}$ and $H E_{1 F}$ respectively represent the initial value and final value of the cognition factor, Formula 24 gives the adjustment formula for $H E_{1}$ :

$$
H E_{1}=H E_{1 C}+\left(H E_{1 F}-H E_{1 F}\right) * \frac{(\psi-\phi)}{\psi}
$$

According to above formula, $H E_{1}$ showed a decreasing trend; assuming $H E_{2 C}$ and $H E_{2 F}$ respectively represent the initial value and final value of the social factor, Formula 25 gives the adjustment formula for $\mathrm{HE}_{2}$ :

$$
H E_{2}=H E_{2 C}+\left(H E_{2 F}-H E_{2 F}\right) * \frac{\phi}{\psi}
$$

According to above formula, $H E_{2}$ showed an increasing trend.

Parameters of the neural network such as $\theta, H E_{i j}$, and $\varepsilon_{i j}$ that need to be optimized were coded in matrix form, then, each particle in the improved PSO can be expressed as:

$$
D(i)=[\theta, H E, \varepsilon]
$$

Figure 3 shows the implementation flow of the FNN optimized by the improved PSO. 

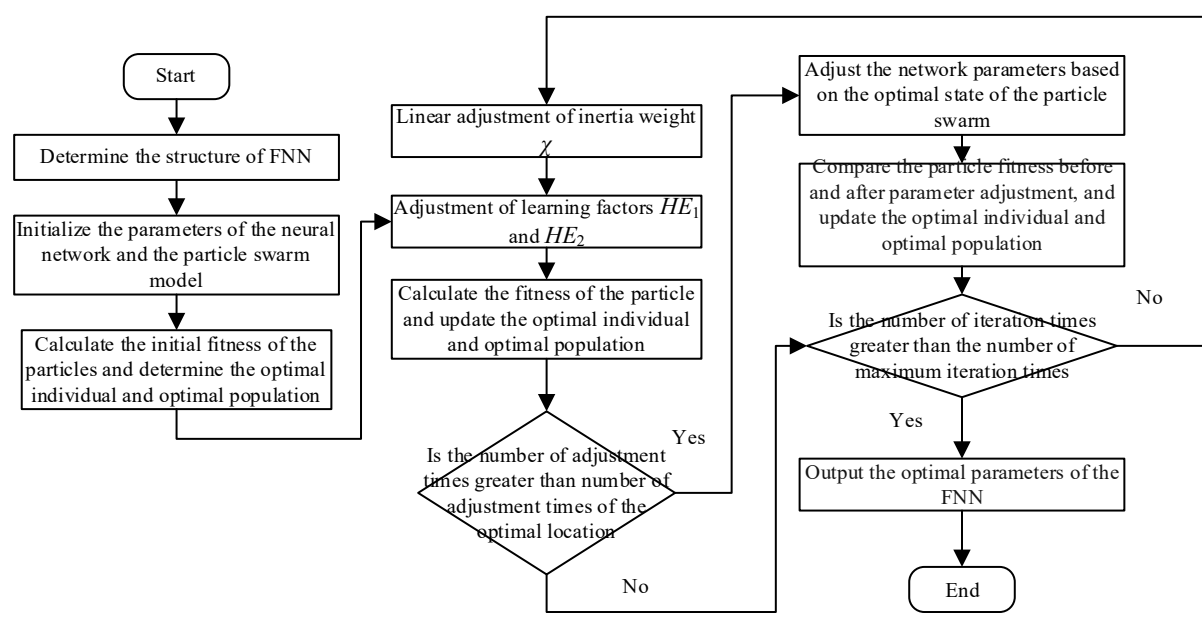

Fig. 3. Implementation flow of the FNN optimized by the improved PSO

\section{$5 \quad$ Experimental results and analysis}

To ensure certain reliability and validity of the constructed EIS, this paper used a commonly-recognized reliability analysis method to analyze the data of the EIS. Table 1 gives the reliability test results of the constructed EIS. According to the table, the Cronbach's $\alpha$ values of the selected evaluation indexes of the I\&E ability of computer majors were all greater than 0.80 , and the total correlation coefficients of the corrected terms were also greater than 0.5 .

In the simulation experiment designed in this study, a 5-dimensional FNN model structure was selected, that is, the error and error change rate between the predicted values and the actual values of the evaluation scores of the five first-level indexes (namely innovation ability, professional quality, entrepreneurial resource integration, team organization and management, and entrepreneurial risk-taking) were selected, then, by converting the quantified input factors of the two to the fuzzy theory domain, 5 fuzzy language variables were obtained, Figure 4 gives a schematic diagram of the selected membership function.

To verify the optimization effect of the improved PSO on the FNN, this study designed an experiment to compare the traditional PSO and the improved PSO. Suppose: the population size of the particle swarm was 100 , the inertia weight $\chi$ was set to 0.9 , and the initial values of the cognition factor $H E_{1}$ and the social factor $H E_{2}$ were set to 2.5 and 0.5 , respectively. In the improved PSO, with the increase of the particle update times, parameters $\chi, H E_{1}$ and $H E_{2}$ were adjusted gradually, the value of $\chi$ was decreased to 0.4 , the value of $H E_{1}$ was decreased to 0.5 , and the value of $H E_{2}$ was increased to 2.5. The maximum numbers of adjustment times and iteration times of the PSO were respectively set to be 10 and 200, and the maximum number of iteration times of the neural network was set to be 1000. Figure 5 and Figure 6 give the change 
Paper-Evaluation of Innovation and Entrepreneurship Ability of Computer Majors based on Computer...

curves of the training performance index of the FNNs improved by the traditional PSO and the improved PSO.

Table 1. Reliability test results of the EIS

\begin{tabular}{|c|c|c|c|c|}
\hline $\begin{array}{l}\text { First-level } \\
\text { index }\end{array}$ & $\begin{array}{c}\text { Second-level } \\
\text { index }\end{array}$ & $\begin{array}{c}\text { Total correlation of } \\
\text { corrected terms }\end{array}$ & $\begin{array}{l}\text { Crenbach's } \alpha \text { reliability } \\
\text { coefficient after deletion }\end{array}$ & $\begin{array}{c}\text { Crenbach's } \alpha \\
\text { reliability coefficient }\end{array}$ \\
\hline \multirow{3}{*}{$I E_{1}$} & $\mathrm{IE}_{11}$ & 0.773 & 0.879 & \multirow{3}{*}{0.824} \\
\hline & $\mathrm{IE}_{12}$ & 0.767 & 0.795 & \\
\hline & $\mathrm{IE}_{13}$ & 0.752 & 0.791 & \\
\hline \multirow{8}{*}{$I E_{2}$} & $\mathrm{IE}_{21}$ & 0.773 & 0.879 & \multirow{8}{*}{0.824} \\
\hline & $\mathrm{IE}_{22}$ & 0.767 & 0.795 & \\
\hline & $\mathrm{IE}_{23}$ & 0.752 & 0.791 & \\
\hline & $\mathrm{IE}_{24}$ & 0.685 & 0.808 & \\
\hline & $\mathrm{IE}_{25}$ & 0.712 & 0.859 & \\
\hline & $\mathrm{IE}_{26}$ & 0.605 & 0.703 & \\
\hline & $\mathrm{IE}_{27}$ & 0.704 & 0.814 & \\
\hline & $\mathrm{IE}_{28}$ & 0.608 & 0.815 & \\
\hline \multirow{3}{*}{$I E_{3}$} & $\mathrm{IE}_{31}$ & 0.715 & 0.806 & \multirow{3}{*}{0.845} \\
\hline & $\mathrm{IE}_{32}$ & 0.756 & 0.781 & \\
\hline & $\mathrm{IE}_{33}$ & 0.734 & 0.815 & \\
\hline \multirow{4}{*}{$I E_{4}$} & $\mathrm{IE}_{41}$ & 0.718 & 0.862 & \multirow{4}{*}{0.836} \\
\hline & $\mathrm{IE}_{42}$ & 0.695 & 0.786 & \\
\hline & $\mathrm{IE}_{43}$ & 0.716 & 0.794 & \\
\hline & $\mathrm{IE}_{44}$ & 0.523 & 0.836 & \\
\hline \multirow{4}{*}{$I E_{5}$} & $\mathrm{IE}_{51}$ & 0.653 & 0.736 & \multirow{4}{*}{0.863} \\
\hline & $\mathrm{IE}_{52}$ & 0.708 & 0.825 & \\
\hline & $\mathrm{IE}_{53}$ & 0.776 & 0.881 & \\
\hline & $\mathrm{IE}_{54}$ & 0.634 & 0.811 & \\
\hline
\end{tabular}

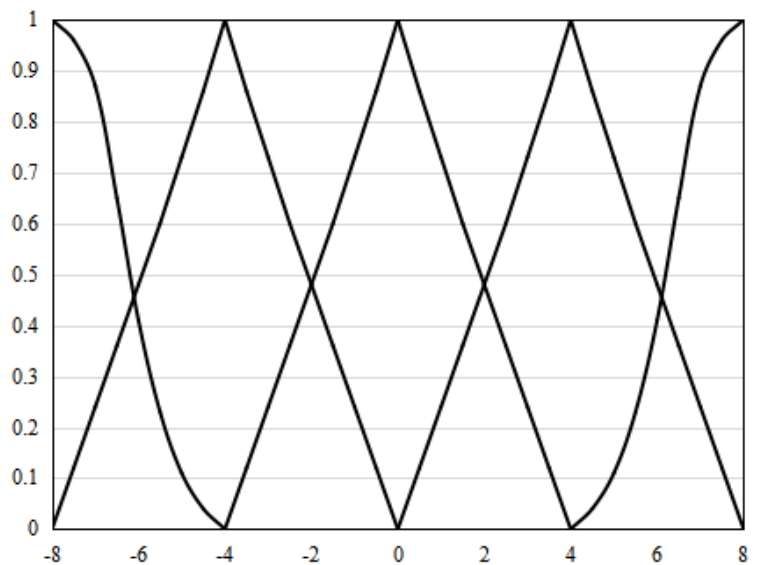

Fig. 4. A schematic diagram of the selected membership function 
Paper-Evaluation of Innovation and Entrepreneurship Ability of Computer Majors based on Computer...

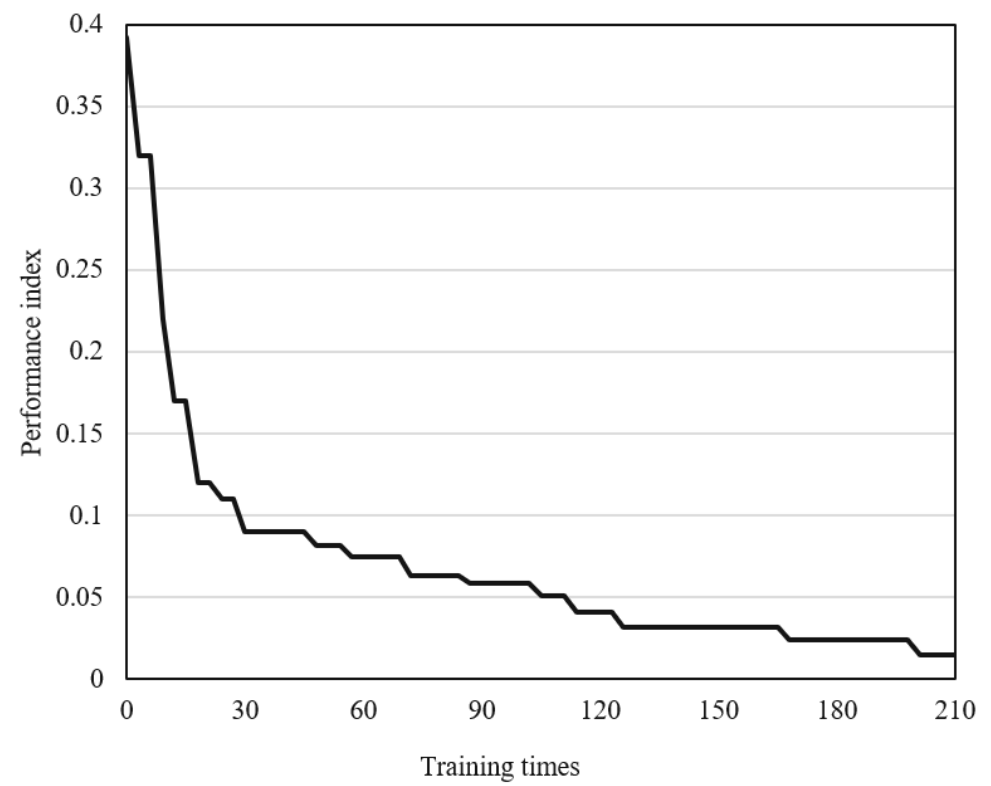

Fig. 5. Change curve of the training performance index of FNN optimized by the traditional PSO

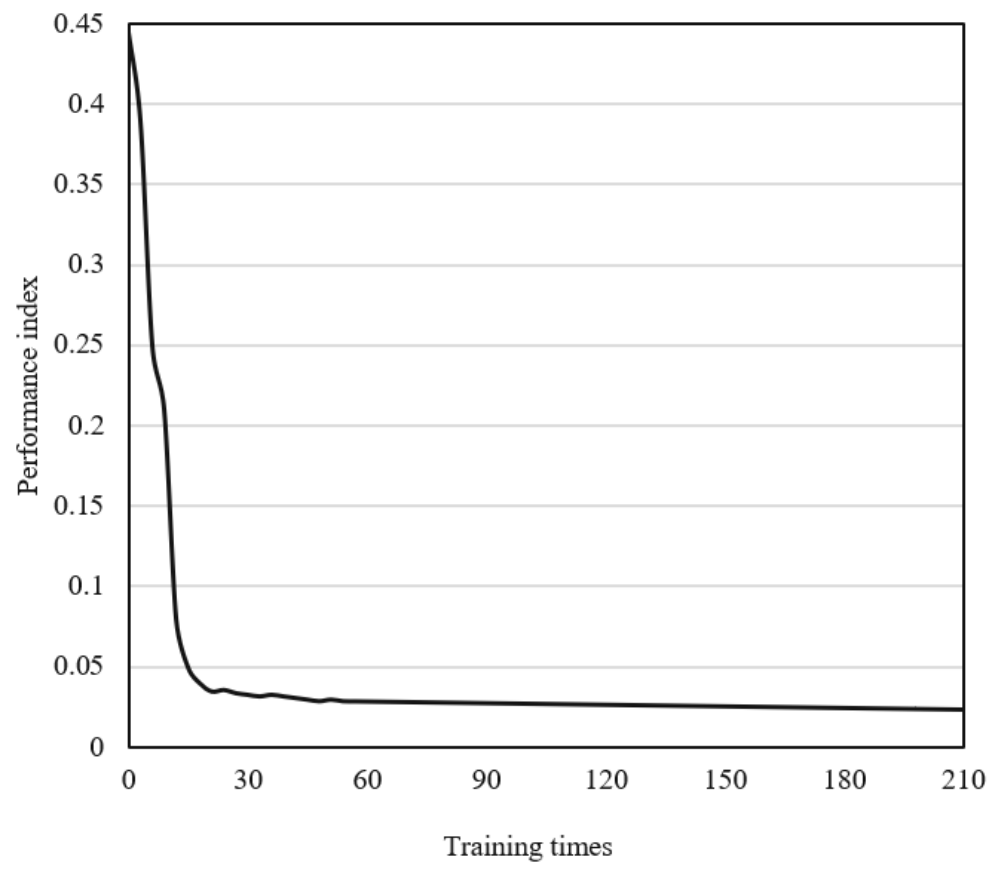

Fig. 6. Change curve of the training performance index of FNN optimized by the improved PSO 
By comparing Figure 5 and Figure 6, we can see that the value of the training performance index of the FNN optimized by the improved PSO changed from about 0.03 before optimization to about 0.01 after optimization, and the overall convergence trend of the performance index curve of the network optimized by the improved PSO was better. Therefore, in the subsequent experiment on the evaluation effect of the I\&E ability of computer majors, the parameters after the optimization of the improved PSO were set to be the initial values of the neural network. Figure 7 shows the fitness curve of parameter optimization of the improved PSO in the model evaluation process.

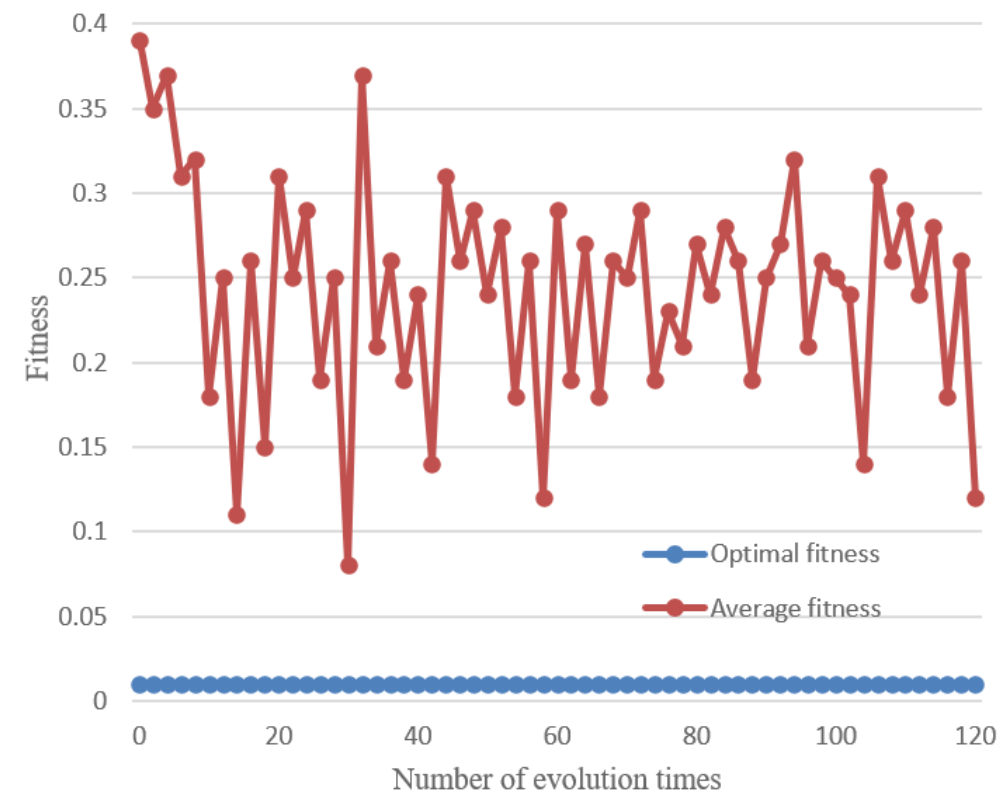

Fig. 7. Fitness curve of parameter optimization of the improved PSO

Figure 8 compares the predicted results of the training set of the evaluation model and the subjective evaluation values of experts. According to the figure, there's not much difference in the predicted evaluation values and the subject evaluation values, the root mean square error (RMSE) value was 0.0014 , the $\mathrm{R}^{2}$ value was 0.9754 , so the predicted results were relatively ideal. Table 2 lists the model training errors of 15 student samples, which had further verified the effectiveness of the model in evaluating the I\&E ability of computer majors. 
Paper-Evaluation of Innovation and Entrepreneurship Ability of Computer Majors based on Computer...

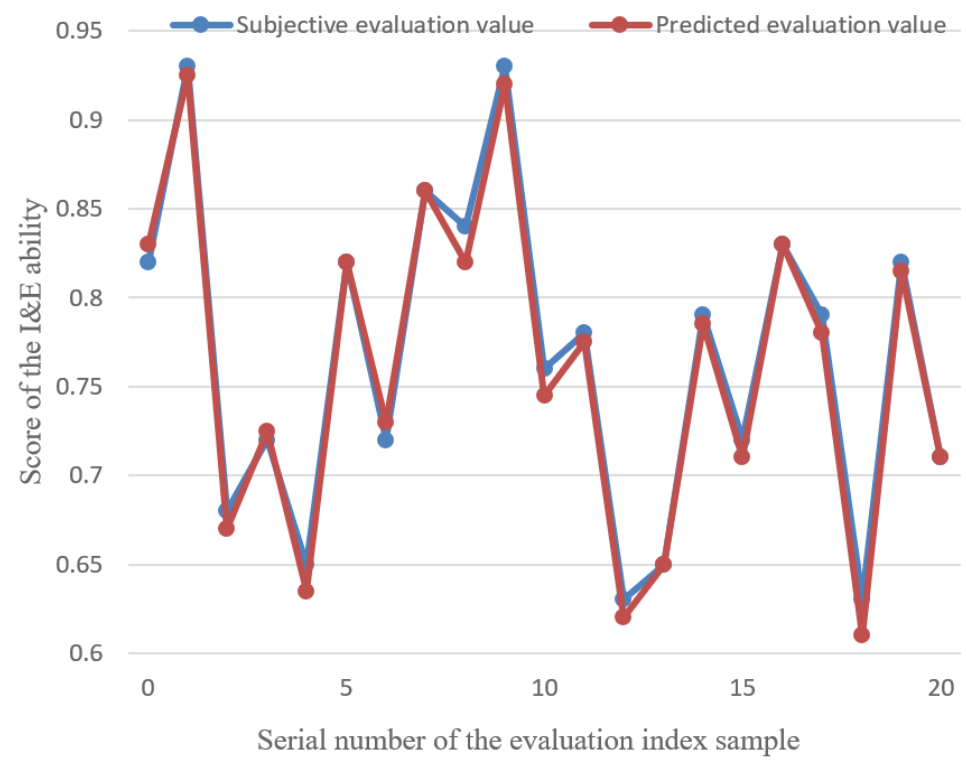

Fig. 8. Comparison of the predicted results of the training set and the subjective evaluation values of experts

Table 2. Model training errors

\begin{tabular}{|l|c|c|c|c|}
\hline $\begin{array}{l}\text { Serial number of } \\
\text { students }\end{array}$ & Actual value & Predicted value & Absolute error & Relative error \\
\hline ST1 & 0.8285 & 0.8275 & 0.0009 & $0.0942 \%$ \\
\hline ST2 & 0.7762 & 0.7751 & 0.0032 & $0.3794 \%$ \\
\hline ST3 & 0.7005 & 0.7019 & 0.0008 & $0.0957 \%$ \\
\hline ST4 & 0.9023 & 0.8924 & 0.0053 & $0.4531 \%$ \\
\hline ST5 & 0.7256 & 0.7243 & 0.0021 & $0.2673 \%$ \\
\hline ST6 & 0.7234 & 0.7295 & 0.0015 & $0.1594 \%$ \\
\hline ST7 & 0.8156 & 0.8172 & 0.0006 & $0.0842 \%$ \\
\hline ST8 & 0.6682 & 0.6735 & 0.0198 & $1.0795 \%$ \\
\hline ST9 & 0.8264 & 0.7167 & 0.0029 & $0.3274 \%$ \\
\hline ST10 & 0.7298 & 0.7134 & 0.0027 & $0.3989 \%$ \\
\hline ST11 & 0.7135 & 0.7056 & 0.0015 & $0.1627 \%$ \\
\hline ST12 & 0.8199 & 0.8191 & 0.0021 & $0.2475 \%$ \\
\hline ST13 & 0.8642 & 0.8692 & 0.0002 & $0.0164 \%$ \\
\hline ST14 & 0.6791 & 0.6678 & 0.0054 & $0.7563 \%$ \\
\hline ST15 & 0.8246 & 0.8315 & 0.0043 & $0.5572 \%$ \\
\hline
\end{tabular}




\section{Conclusion}

This paper researched the I\&E ability of computer majors based on neural network optimized by PSO. In the paper, an EIS of 22 second-level indexes under 5 first-level indexes was designed to evaluate the I\&E ability of college computer majors, and a corresponding FNN evaluation model was established. Then, the optimization method of FNN based on the improved PSO was elaborated, the reliability test results of the EIS were given through experiment, and the reliability and effectiveness of the constructed EIS were verified. Moreover, the paper also gave the change curves of the training performance index of the FNNs optimized by the traditional PSO and the improved PSO, and the results suggested that the overall convergence trend of the performance index curve of the network optimized by the improved PSO was better. At last, this paper compared the predicted results of the training set of the evaluation model and the subjective evaluation values given by the experts, which had further verified the effectiveness of the model in evaluating the I\&E ability of computer majors.

\section{$7 \quad$ References}

[1] Zhang, Y., Hu, M. (2021). Research on the Relationship between Data Empowerment and Service Innovation Capability of Logistics Platform Enterprise. Mathematical Problems in Engineering, 2021: Article ID 9974585. https://doi.org/10.1155/2021/9974585

[2] Hermawati, A., Gunawan, E. (2020). The implementation of dynamic capabilities for small and medium-sized enterprises in creating innovation. VINE Journal of Information and Knowledge Management Systems, 51(1): 92-108. https://doi.org/10.1108/vjikms-08-2019$\underline{0121}$

[3] Wang, L., Wang, K. (2009). The multilevel gray evaluation of innovation capability of Hub-and-Spoke enterprise clusters. In 2009 International Conference on Information Management, Innovation Management and Industrial Engineering, 4: 254-257. https://doi. org/10.1109/iciii.2009.522

[4] Sang, B. (2021). Innovation of enterprise technology alliance based on BP neural network. Neural Computing and Applications, 33(3): 807-820. https://doi.org/10.1007/s00521-02005254-2

[5] Somboonsak, P. (2020). Development Innovation to Predict Dengue Affected Area and Alert People with Smartphones, International Journal of Online and Biomedical Engineering, 16(2): 62-79. https://doi.org/10.3991/ijoe.v16i02.12425

[6] Ma, J., Guo, M. (2020). Analysis of scientific and technological innovation influence factors affect enterprise performance. International Journal of Internet Manufacturing and Services, 7(1-2): 162-175. https://doi.org/10.1504/IJIMS.2020.105031

[7] Smith, C., Fixson, S.K., Paniagua-Ferrari, C., Parise, S. (2017). The Evolution of an Innovation Capability: Making Internal Idea Competitions Work in a Large Enterprise One firm evolved its idea competitions into a broad innovation management system. ResearchTechnology Management, 60(2): 26-35. https://doi.org/10.1080/08956308.2017.1276388

[8] Lin, P., Zhang, X., Yan, S., Jiang, Q. (2020). Dynamic Capabilities and Business Model Innovation of Platform Enterprise: A Case Study of DiDi Taxi. Scientific Programming, 2020: Article ID 8841368. https://doi.org/10.1155/2020/8841368 
[9] Omoseni O.A., Nnamdij, I. N. (2020). Engineering Students' Innovation Competence: A Comparative Analysis of Nigeria and South Africa, International Journal of Engineering Pedagogy, 10(6): 147-155

[10] Zhang, L.Y., Cao, C.Z. (2020). Control power of senior executive, business environment and entrepreneurship. International Journal of Sustainable Development and Planning, 15(7): 1127-1136. https://doi.org/10.18280/ijsdp.150717

[11] Qu, S., Shi, H., Zhao, H., Yu, L., Yu, Y. (2021). Research on Enterprise Business Model and Technology Innovation Based on Artificial Intelligence. Eurasip Journal on Wireless Communications and Networking, 2021(1): 145. https://doi.org/10.1186/s13638-02102025-y

[12] Şahin, T., Riedel, L., Inkermann, D., Wilgeroth, D., Asghari, R., Vietor, T. (2019). How to Foster Innovation? A Methodology to Identify Fields for Fostering Innovation Capability in Small and Medium-Sized Enterprises. In Proceedings of the Design Society: International Conference on Engineering Design, 1(1): 2269-2278. https://doi.org/ $\underline{10.1017 / \text { dsi.2019.233 }}$

[13] Wu, Q., Wang, W. (2019). An empirical analysis on the forming mechanism of the innovation capability of service-oriented manufacturing enterprises. International Journal of Manufacturing Technology and Management, 33(3-4): 189-218. https://doi.org/10. $\underline{1504 / \mathrm{ijm} t \mathrm{~m} .2019 .10022679}$

[14] Boscoianu, M., Prelipcean, G., Lupan, M. (2018). Innovation enterprise as a vehicle for sustainable development-A general framework for the design of typical strategies based on enterprise systems engineering, dynamic capabilities, and option thinking. Journal of Cleaner Production, 172: 3498-3507. https://doi.org/10.1016/j.jclepro.2017.06.120

[15] Saddhono, K., Hasanudin, C., Fitrianingsih, A. (2019). The ability to think creatively on SSCS using schoology Apps, how is the student's language metacognitive awareness? Ingénierie des Systèmes d'Information, 24(4): 367-375. https://doi.org/10.18280/isi. $\underline{240402}$

[16] Su, Y., Yu, Y.Q. (2019). Spatial interaction network structure and its influence on new energy enterprise technological innovation capability: Evidence from China. Journal of Renewable and Sustainable Energy, 11(2): 025902. https://doi.org/10.1063/1.5043618

[17] Louw, L., Essmann, H.E., Du Preez, N.D., Schutte, C.S.L. (2017). Architecting the enterprise towards enhanced innovation capability. South African Journal of Industrial Engineering, 28(4): 50-65. https://doi.org/10.7166/28-4-1628

[18] Chen, P. (2014). Enterprise Technology Innovation Capability Evaluation Based on Knowledge Management. In 2014 Seventh International Joint Conference on Computational Sciences and Optimization, 280-283. https://doi.org/10.1109/cso.2014.140

[19] Kim, M. K., Park, J. H., Paik, J. H. (2018). Factors influencing innovation capability of small and medium-sized enterprises in Korean manufacturing sector: Facilitators, barriers and moderators. International Journal of Technology Management, 76(3-4): 214-235. https://doi.org/10.1504/ijtm.2018.10012461

[20] Xu, Y., Ma, J., Lu, Y. (2015). Innovation catch-up enabled by the window of opportunity in high-velocity markets and the intrinsic capabilities of an enterprise: the case of HTC. International Journal of Technology Management, 69(2): 93-116. https://doi.org/10.1504/ ijtm.2015.071550

[21] Ran, R., Wang, B. J. (2015). Combining grey relational analysis and TOPSIS concepts for evaluating the technical innovation capability of high technology enterprises with fuzzy information. Journal of Intelligent \& Fuzzy Systems, 29(4): 1301-1309. https://doi.org/10. $\underline{3233 / \text { ifs- } 141380}$ 
Paper-Evaluation of Innovation and Entrepreneurship Ability of Computer Majors based on Computer...

[22] Li, W.H. (2014). Structural Analysis of Factors Influencing Quality Innovation Capability in Manufacturing Enterprises. In Key Engineering Materials, 584: 268-276. https://doi.org/ 10.4028/www.scientific.net/KEM.584.268

[23] Di, R., Xu, M., Wang, Y. (2014). Research on evaluation capability and mode selection of radical technological innovation in service enterprises. In 2014 International Conference on Management Science \& Engineering 21th Annual Conference Proceedings, 1753-1758. https://doi.org/10.1109/icmse.2014.6930446

[24] Tu, Z.Z., Gu, X. (2013). Fuzzy integral evaluation on team innovation capability based on team interactive process - Take enterprise's new product R\&D team as case study. BioTechnology: An Indian Journal, 8(3): 367-373.

[25] Kowal, J., Mäkiö, J., Jasińśka-Biliczak, A. (2017). Business competencies and innovation capability in cross-border small regional enterprises. In 2017 IEEE 15th International Conference on Industrial Informatics (INDIN), 905-910. https://doi.org/10.1109/indin.2017. $\underline{8104892}$

[26] Collinson, S.C., Wang, R. (2012). The evolution of innovation capability in multinational enterprise subsidiaries: Dual network embeddedness and the divergence of subsidiary specialisation in Taiwan. Research Policy, 41(9): 1501-1518. https://doi.org/10.1016/j. $\underline{\text { respol.2012.05.007 }}$

[27] Bai, Z., Li, X., Zhang, X. (2016). Empirical Research on Influence Factors of Enhancing Independent Innovation Capability for Small and Medium-sized Technology-Based Enterprises. International Journal of Simulation--Systems, Science \& Technology, 17(25): 11.111.6. https://doi.org/10.5013/ijssst.a.17.31.11

\section{Authors}

Li Ding, master and lecturer of Information Engineering Department, Hebei Vocational University of Technology and Engineering, majoring in software technology; published more than 10 academic papers in recent years, 2 of which were published by Chinese core journals; obtained 2 utility model patents and 5 software copyrights.

Xuguang Chai, master and associate professor of Information Engineering Department, Hebei Vocational University of Technology and Engineering, majoring in big data technology and application; published more than 20 academic papers in recent years, of which 4 were published by EI journals and 2 were published by Chinese core journals; obtained 1 invention patent, 7 utility model patents, and 5 software copyrights (Email: chaixuguang@163.com)

Fanjin Zeng, master and lecturer of Information Engineering Department, Hebei Vocational University of Technology and Engineering, majoring in big data technology and application, published 5 academic papers in recent years; obtained 2 utility model patents and 3 software copyrights (Email: 690860941@qq.com).

Article submitted 2021-08-15. Resubmitted 2021-09-12. Final acceptance 2021-09-14. Final version published as submitted by the authors. 